\title{
Post-Pandemic Activity Rebounds, but Many Remain Outside the Labor Force
}

\author{
Nathan Jefferson, Associate Business Economist
}

W

hat's the current state of the labor market? If you look at the most widely reported employment indicators, it may appear that there's a good deal of slack: Total nonfarm payroll employment rose by a lower-than-expected 269,000 jobs in April and 583,000 in May, and the total number of employed workers remains more than 6.7 million below its February 2020 peak. But the economy recorded a record-high 9.2 million job openings in May 2021, which aligns with widespread anecdotal reports from business owners who are struggling to meet staffing needs-signs of a tight labor market.

Jobs are returning, but the pandemic has accelerated longtime trends by knocking millions of people out of the labor force.

Here's the challenge in trying to parse out the exact state of the labor market: Jobs are returning, ${ }^{1}$ but the pandemic has accelerated longtime trends by knocking millions of people out of the labor force. Some of these exits were retirements, but many were prime-age workers previously employed in industries heavily affected by the pandemic.
Most workers who left the labor force were women, ${ }^{2}$ which reflects the domestic burden imposed by lockdowns. For these workers, barriers remain to rejoining the labor force even as economic activity begins to pick back up.

\section{Unemployment Rate Excludes an Important Group}

The headline unemployment rate measures the share of people who are actively looking for work and unable to find it. That first part is important: Many Americans are out of the labor force for any number of reasons. Because they're not actively looking for work, they aren't considered unemployed. Students and retirees, for example, make up a large chunk of this population.

There are also larger demographic trends at play. As the workforce has aged in recent decades, the average participation rate has consistently declined. ${ }^{3}$ The figure shows that the participation rate has fallen from above $67 \%$ shortly after 2000 to under $63 \%$ in 2019 . Of course, the pandemic's effects are still evident: More than 8 million workers left the labor force between February and May 2020.

While economic conditions have improved, the labor force has been slower to rebound. As of April's BLS report, the number of people not in the labor force was 5 million greater than pre-pandemic levels.

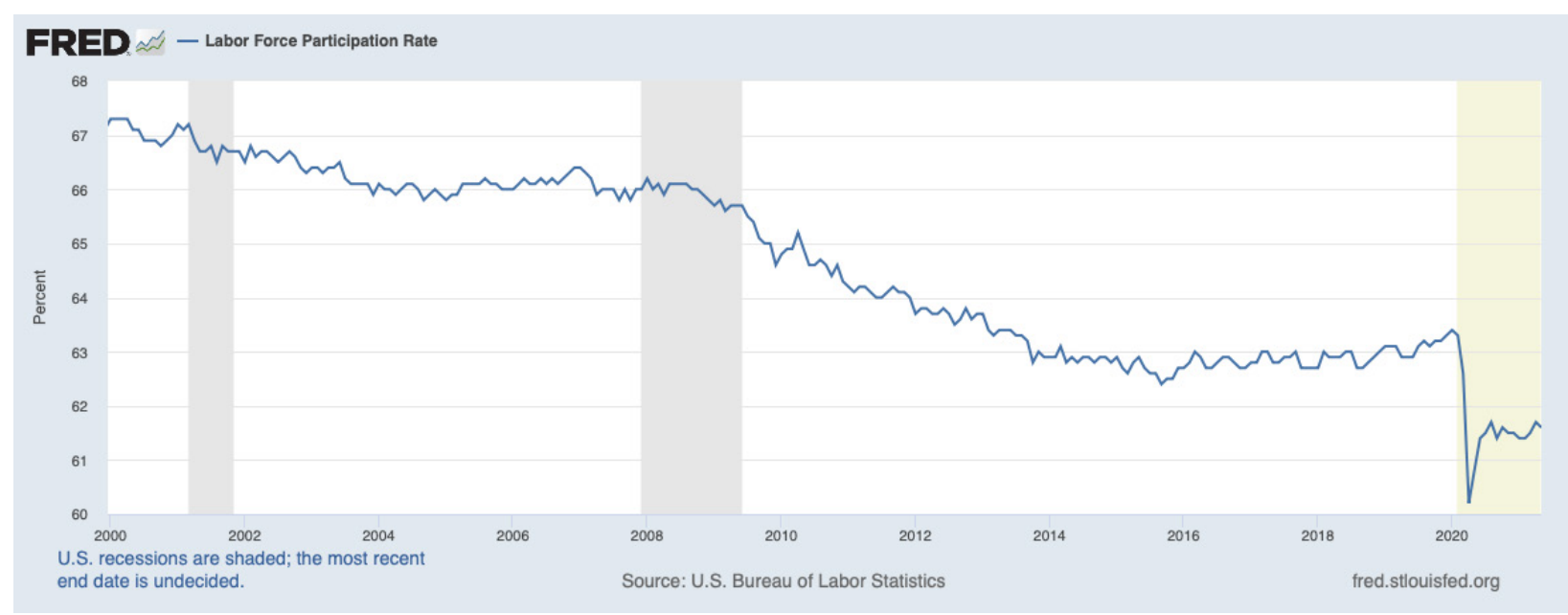

SOURCE: FRED ${ }^{\oplus}$, Federal Reserve Bank of St. Louis; https://fred.stlouisfed.org/graph/?g=F4xn. 


\section{Exit Trends}

A worker leaving the labor force isn't necessarily evidence of a weak labor market-someone retiring, for example, adds to that total. It's useful to look at a more precise decomposition of labor force data so that we can see if any trends are evident.

What do the data on the prime-working-age population suggest? BLS data show that the share of 25- to 54-yearolds in the labor force dropped from $82.9 \%$ in February 2020 to $79.8 \%$ in April 2020. A year later, it's partially recovered to $81.3 \%$.

It's also useful to look at industry-specific data: March 2021 CPS data show that individuals who were employed in Q1 of 2020 but currently out of the labor force were disproportionately in the transport, hospitality, and food services sectors. It's not a surprise that those sectors are overrepresented in job loss data; they were some of the ones hardest hit by the pandemic.

\section{Gender}

Women make up the majority of those who left the labor force, which is a reversal of the trend of a rising labor force participation rate for women over the past few decades. Prior to this crisis, prime-age women were less likely than men to leave the workforce: While the share of prime-age men in the labor force fell 2.6\% between 1999 and 2019, the share of prime-age women fell just $0.8 \%$.

That's changed over the past year. In March 2021, 74.1\% of prime-age women were in the labor force, down from $77.1 \%$ in February 2020. Evidence points to increased domestic responsibilities being one of the primary reasons for this shift. Women face greater caregiving responsibili$\underline{\text { ties, }}$, and the first few months of the pandemic saw mothers decrease their work hours four to five times more than fathers.

\section{Frictions May Take Time to Resolve}

Two key points help clarify what's currently happening in U.S. labor markets. First, the demand side of the equation has rebounded faster than the supply side, with GDP returning to pre-pandemic trends. Second, the economic shocks of the past year led many to leave the labor market.

While improving economic conditions are alleviating these frictions, they won't disappear overnight. The May Survey of Professional Forecasters projects monthly job growth of 331,600 jobs per month in 2021 and 405,100 in 2022. Although this is up 30\% from three months ago, it would take the economy almost two years to reach prepandemic employment levels at this pace.

\section{Notes}

\footnotetext{
${ }^{1}$ Measures of economic conditions like the Kansas City Fed's LCMI have shown significant increases in demand for labor, and the ratio of unemployed persons per job opening has returned to 2017 levels.

2 Net 2.4 million women left the labor force from February 2020 to February 2021, compared with 1.8 million men over the same period (IPUMS-CPS, University of Minnesota; www.ipums.org).

${ }^{3}$ Aaronson et al. argue that the causes of this recent decline are structural.
} 